\title{
La competencia como punto de partida y no como finalidad del proceso de enseñanza en ciencias
}

\author{
Mirada de la Enseñanza de las Ciencias basada en Competencias en el contexto colombiano a \\ la luz de la redescripción representacional y la teoría de los campos conceptuales
}

\section{Competence as a starting point and not the end in the process in the teaching of Sciences \\ Looking at Science Teaching Competency based in the Colombian context in light of representational redescription $R R$ and conceptual fields theory TCC}

\author{
Juan Samuel Rangel Luengas \\ juansamueldj@gmail.com
}

\begin{abstract}
Resumen
El siguiente escrito se propone, en primer lugar, relacionar la teoría de los Campos Conceptuales $\boldsymbol{T C C}$, de Gérard Vergnaud, con el modelo de Redescripción Representacional $\boldsymbol{R} \boldsymbol{R}$ de Karmiloff-Smith y luego, ubicar las coincidencias de estas teorías en el escenario de la enseñanza por competencias en el contexto colombiano. Es el resultado de la reflexión de la lectura de documentos sobre campos conceptuales y el libro del Karmiloff-Smith, llamado más allá de la modularidad, esta reflexión planteada al interior del contexto colombiano. Para tal fin, se presenta una breve descripción de las teorías y algunas definiciones de competencia vigentes en Colombia, particularmente en el área matemática, aunque esta mirada no difiere de la propuesta para las demás ciencias básicas. Finalmente, el punto de intersección o compatibilidad entre las teorías, que aplicadas a la enseñanza por competencias, tal como se realiza actualmente en Colombia, permite plantear la siguiente hipótesis: Es un error en la enseñanza en ciencias, considerar la competencia como punto final del proceso educativo, por el contrario, la competencia adquiere la característica de estado inicial en el camino hacia la formación de conocimiento científico.
\end{abstract}

Palabras claves: Teoría de los campos conceptuales. Redescripción representacional. Competencia. Enseñanza de las ciencias.

\section{Introducción}

Actualmente, la enseñanza por competencias es, en el contexto colombiano, una directriz reglamentada desde la política educativa. Los currículos están construidos desde esta perspectiva y es prioridad que los estudiantes alcancen la competencia en diferentes áreas relacionadas con las ciencias. Así, la competencia es una finalidad en todas áreas de conocimiento, son el norte a seguir

“... la expresión ser matemáticamente competente...Esta noción ampliada de competencia está relacionada con el saber qué, el saber qué hacer y el saber cómo, cuándo y por qué hacerlo. Por tanto, la precisión del sentido de estas expresiones 
implica una noción de competencia estrechamente ligada tanto al hacer como al comprender. Si bien es cierto que la sociedad reclama y valora el saber en acción o saber procedimental, también es cierto que la posibilidad de la acción reflexiva con carácter flexible, adaptable y generalizable exige estar acompañada de comprender qué se hace y por qué se hace y de las disposiciones y actitudes necesarias para querer hacerlo, sentirse bien haciéndolo y percibir las ocasiones de hacerlo.”( MEN 2006) ${ }^{1}$

Como puede apreciarse, la competencia es un punto a llegar en la formación de los estudiantes y está muy ligada al hacer, tal como lo manifiesta la anterior definición.

Sin embargo, el hacer es importante, pero hace falta avanzar hacia la verbalización y con el fin de acercar a los estudiantes al trabajo científico, a la validación del conocimiento. Esta idea tiene su sustento epistemológico en los programas de investigación científica. La teoría científica, como un conjunto de hipótesis o núcleo fuerte que aunque se percibe irrefutable, está rodeado, o al menos eso se espera, de teorías que luego pueden reclamar su espacio como verdaderas. No existen verdades absolutas en ciencias, y algunas actuaciones que ahora se aceptan como válidas, pueden no serlo desde una nueva teoría con mayor nivel de predicción o explicación de los fenómenos.

Entendida la ciencia de esta manera, reclama una enseñanza de manera similar, en la que los conocimientos sean validados, sin presentar verdades irrefutables o inmóviles, sino que, tiene como dinámica, estar sujetas a procesos de falsación. Desde esta mirada la enseñanza en ciencias requiere que los estudiantes se involucren presentando, elaborando y verbalizando sus teorías, no solo actuando en consecuencia de las mismas. Las teorías en diferentes niveles pueden ser Tanto aquellas que pueden confluir del aprendizaje cotidiano, como las que han sido elaboradas por una comunidad científica con vigencia al interior de la ciencia actual.

Ahora, si se presente cumplir con este objetivo, desde dos de las teorías neopiagetianas, es insuficiente que el estudiante sea competente, se requiere avanzar mucho más y esa es la hipótesis que se defenderá a continuación. Para ello, se presenta una breve explicación de la teoría de los campos conceptuales y la teoría de la redescripción representacional, luego algunas definiciones de competencias particularmente en el área de matemáticas, que tiene vigencia aun en el contexto educativo colombiano, estos apartes los puede omitir quien ya tenga claridad de estos aspectos. Finalmente, se presenta como conclusión la justificación de la hipótesis planteada.

\footnotetext{
${ }^{1}$ ESTÁNDARES BÁSICOS DE COMPETENCIASEN MÁTEMÁTICAS pág. 50.
} 


\section{Teoría de los campos conceptuales}

Una forma de acercarnos a la definición de campo conceptual, es definir concepto. Desde la teoría planteada por Gérard Vergnaud, un concepto adquiere sentido desde situaciones variadas que le permiten su operacionalidad. Así, la definición de concepto no puede estar desligada de lo que se entiende por situación, porque ésta, condiciona la forma de actuar del sujeto, que la enfrenta. Al quedar inmerso en una situación, el individuo hace uso de formas de actuar interiorizadas previamente, que se denominan invariantes operatorios, que también forman parte de la definición de concepto. Así, la definición de concepto, se requiere tener en cuenta tres características simultáneamente

\section{“... un concepto es una tripleta de tres conjuntos: $C(S, I, \Gamma)$}

S: conjunto de situaciones que dan sentido al concepto (la referencia)

I: conjunto de invariantes sobre los cuales reposa la operacionalidad de los esquemas (el significado)

$\Gamma:$ conjunto de las formas lingüísticas y no lingüísticas que permiten representar simbólicamente el concepto, sus propiedades, las situaciones y los procedimientos de tratamiento (el significante)." (Vergnaud: 1990, Pág. 140) ${ }^{2}$

Un concepto requiere de los tres conjuntos a la vez y ninguno reemplaza al otro, son escenarios diferentes, pero entrelazados. El referente, el significado y el significante, que se requieren simultáneamente para apropiar un concepto.

\section{Situación (referente)}

Es entendida como una tarea y no desde el punto de vista de la didáctica. Es importante resaltar que una situación es compleja si se puede subdividir en tareas de menor complejidad, entendiendo que, cada tarea tiene su propia dificultad y la suma de las dificultades no es la dificultad total, tal que, si alguna de ellas no se realiza correctamente la tarea global carece de éxito.

Una situación no es exclusiva para un concepto, porque en ella intervienen varios de ellos. Las situaciones, permiten establecer relaciones entre conceptos de distinta naturaleza y observar al

\footnotetext{
${ }^{2}$ La negrilla no es del texto original.
} 
individuo en acción según se lo exigen. Por ésta razón, las situaciones se convierten en el escenario motivador de la conceptualización. Aunque una situación no involucra un solo concepto, si es posible que para aun grupo o clase de situaciones, el invariante usado por el sujeto sea el mismo, por ello, es necesario ampliar el repertorio de esquemas que acostumbran a utilizar los estudiantes.

\section{Esquema: invariantes operatorios (significado)}

El esquema, contiene reglas para regular las acciones, cuyo orden de aplicación, puede variar, porque dependen de la situación. El esquema, necesita algunas consideraciones adicionales,

...permite de generar una clase de conductas diferentes en función de las características particulares de cada una de las situaciones de la clase a la cual se dirige. Esto es posible porque el esquema comporta:

- invariantes operatorios (conceptos-en-acto y teoremas-en-acto) que pilotan el reconocimiento por el sujeto de los elementos pertinentes de la situación, y la recogida de información sobre la situación a tratar;

- anticipaciones del fin a lograr, de los efectos a esperar y de las etapas intermedias eventuales;

- reglas de acción del tipo si... entonces... que permiten generar la serie de acciones del sujeto;

- inferencias (o razonamientos) que permiten "calcular" las reglas y las anticipaciones a partir de las informaciones y del sistema de invariantes operatorios de los que dispone el sujeto. (ibíd. Pág.15)

La teoría de los campos conceptuales es neo constructivista, influenciada en gran medida por los planteamientos de Jean Piaget, por tal razón, es muy importante para la TCC, el concepto de esquema, que determina el comportamiento del sujeto, quedan a la vista diferentes herramientas con las que cuenta para enfrentarse a diferentes tipos de situaciones.

En la interacción situación-esquema, puede ocurrir que un individuo tenga los esquemas necesarios para afrontar una situación en cuyo caso la aplicación se realizará de forma automatizada. Pero, por otra parte, puede ocurrir que no tenga los esquemas necesarios para 
avanzar y en este caso tendrá que poner a prueba su creatividad. Al interior del esquema, se encuentran los invariantes operatorios: teoremas en acto y conceptos en acto (el significado).

El funcionamiento cognitivo del sujeto en situación depende del estado de sus conocimientos, implícitos o explícitos. Es necesario por tanto conceder una gran atención al desarrollo cognitivo, a sus continuidades, a sus rupturas, a los pasos obligados, a la complejidad relativa de las clases de problemas, procedimientos, representaciones simbólicas, al análisis de los principales errores y de los principales descubrimientos. (ibíd. Pág.20-21)

Finalmente, teniendo los elementos básicos del concepto, es posible definir qué se entiende por Campo Conceptual. En términos generales, es un conjunto de situaciones que ponen en juego varios conceptos, relaciones entre ellos y diferentes representaciones que mantienen relación estrecha (Vergnaud, G. 1990 Pág. 140), dicho en otras palabras,

“... un conjunto informal y heterogéneo de problemas, situaciones, conceptos, relaciones, estructuras, contenidos y operaciones de pensamiento conectadas unas con otras y susceptibles de ser entretejidas durante el proceso de adquisición" (Vergnaud, 1982, Pág. 40)

\section{Modelo de re-descripción representacional (significante)}

Karmiloff-Smith plantea en su teoría tres fases que ocurren cíclicamente y vuelven a repetirse de forma periódica: En la primera, la relación del niño con el entorno del cual realiza una representación interna, la segunda al interior del sujeto, en esta fase se modifica la conducta y la tercera, como conciliación entre las representaciones y la conducta. Las representaciones internas se presentan en distintos formatos y el modelo RR explica el proceso, definiendo niveles desligados de una edad en particular, que pueden repetirse iterativamente en distinto tiempo. El modelo consta de cuatro niveles denominados como implícito, Explícito 1, Explícito 2, Explícito 3, representados como (I), E(1), E(2) y E(3) respectivamente (Karmiloff-Smith), este es un modelo de fases.

Consiste en un proceso cíclico mediante el cual información ya presente en las representaciones del organismo que funcionan independientemente y están al servicio de propósitos particulares se pone progresivamente a disposición de otras partes de del sistema cognitivo, gracias a la intervención de procesos de redescripción. (Karmiloff-Smith: 1994, pág.37)

En la primera fase, el conocimiento es implícito, no está en un formato disponible para otros operadores del sistema cognitivo, las representaciones se almacenan como procedimientos y que 
se activan ante la interacción con un ente externo. Cada representación nueva, se guarda aparte, no se relacionan entre si y no es posible acceder a ellas de manera consiente.

... la redescripcion representacional es un proceso mediante el cual información que se encuentra implícita en la mente llega a convertirse en conocimiento explicito para la mente, primero dentro de un dominio y posteriormente, a veces a lo largo de diferentes dominios. (ibíd. Pág. 37)

El modelo RR, explica el camino de lo implícito hacia lo explicito, aunque debe hacerse una observación importante, el termino explicito no implica verbalizar.

Luego de superar el nivel de lo implícito, se bosquejan tres niveles en el campo de lo explícito. Estos niveles son: E1 no ofrece la capacidad de acceso consciente, en el nivel E2 las representaciones se hacen accesibles a la conciencia, sin que esto indique que pueden verbalizarse y en el nivel E3 el conocimiento se recodifica en un código común a todos los sistemas, el código está lo suficientemente próximo al lenguaje natural y esto lo hace fácilmente expresable, es decir en este nivel es posible verbalizar el conocimiento. De un nivel a otro lo que ocurre es un recodificación o redescripción de la representación, el nivel E2 es un redescripción del nivel E1 y en consecuencia, el nivel E3 es una redescripción del nivel E2. Siendo cada redescripción una versión más condensada del nivel anterior.

Siempre hay un periodo de maestría conductual que parece ocurrir antes del cambio representacional... un estado estable. (Ibíd. Pág. 223)

Se debe alcanzar un periodo de maestría conductual antes de que se produzca la redescripción y este proceso es de dominio general. Al interior de cada fase, se realizan re descripciones, realizando adiciones representacionales, utilizando la retroalimentación negativa, hasta alcanzar la maestría conductual y luego la iniciativa desde una retroalimentación positiva en un sistema estable. Se requiere la estabilidad para avanzar al siguiente dominio. Debe aclararse que no debe confundirse dominio con modulo. De todas formas la transición que se realiza entre los dominios, es del conocimiento implícito hacia el explícito verbalizado

La mente humana explota su complejidad representacional por medio de la rerepresentación de su conocimiento implícito en formatos explícitos. (Ibíd. Pág. 235)

En las investigaciones con bebes y niños de temprana edad es posible argumentar como se presenta el modelo RR en los diferentes dominios E1, E2 y E3. Pero, esta última etapa para 
edades posteriores, parece no alcanzarse y la estabilidad se conserva en el nivel E2. Así muchos de los conocimientos, no logran esa etapa en la cual el código de representación es común y permite la verbalización.

\section{Competencias}

Aunque es extensa la teoría sobre competencias, en síntesis, en el contexto colombiano, se valida competente a aquel estudiante que posee un saber, que le permite la acción en un contexto particular, se escucha frecuentemente parafrasear a los profesores "es saber-hacer en contexto".

Esta definición informal presenta que el estudiante, dominar un saber, no necesariamente disciplinar sino que puede ser un saber cotidiano no necesariamente aprendido en la escuela. El individuo por medio del hacer, permite evidenciar ese saber ${ }^{3}$, así, la competencia solo es verificable por la acción, un estudiante competente genera acciones ante una situación o problema. Finalmente, es importante el contexto al interior del cual el individuo se desenvuelve. Particularmente en matemáticas,

$$
\begin{aligned}
& \text { “... es entendida como capacidad para realizar adecuadamente tareas matemáticas } \\
& \text { específicas, debe complementarse con la comprensión matemática de las técnicas } \\
& \text { necesarias para realizar las tareas (¿por qué la técnica es adecuada?, ¿cuál es su ámbito } \\
& \text { de validez?) y las relaciones entre los diversos contenidos y procesos matemáticos } \\
& \text { puestos en juego..” (Godino, 2002). }{ }^{4}
\end{aligned}
$$

En esta definición, nuevamente se resalta la necesidad que el logre ejecutar una acción enfocada hacia la solución de problemas

$$
\begin{aligned}
& \text { “... la capacidad de administrar nociones, representaciones y utilizar procedimientos } \\
& \text { matemáticos para comprender e interpretar el mundo real. Esto es, que el alumno tenga } \\
& \text { la posibilidad de matematizar el mundo real, lo que implica interpretar datos; establecer } \\
& \text { relaciones y conexiones; poner en juego conceptos matemáticos; analizar regularidades; } \\
& \text { establecer patrones de cambio; encontrar, elaborar, diseñar y/o construir modelos; } \\
& \text { argumentar; justificar; comunicar procedimientos y resultados." (LLECE, 2005) }
\end{aligned}
$$

Cronológicamente, esta definición está más elaborada y exige del individuo, la capacidad de verbalizar los procedimientos y resultados. Nuevamente los conceptos matemáticos deben emplearse coherentemente en una situación particular.

\footnotetext{
${ }^{3}$ En este aspecto se presenta una incompatibilidad con las teorías neopiagetianas.

${ }^{4}$ Pag 15

${ }^{5}$ Página 15 marco teorico ICFES
} 
“... la expresión ser matemáticamente competente...Esta noción ampliada de competencia está relacionada con el saber qué, el saber qué hacer y el saber cómo, cuándo y por qué hacerlo. Por tanto, la precisión del sentido de estas expresiones implica una noción de competencia estrechamente ligada tanto al hacer como al comprender. Si bien es cierto que la sociedad reclama y valora el saber en acción o saber procedimental, también es cierto que la posibilidad de la acción reflexiva con carácter flexible, adaptable y generalizable exige estar acompañada de comprender qué se hace y por qué se hace y de las disposiciones y actitudes necesarias para querer hacerlo, sentirse bien haciéndolo y percibir las ocasiones de hacerlo."( MEN 2006) ${ }^{6}$

Esta definición amplia las anteriores manifestando que el actuar debe ser pertinente e invitar a la reflexión sobre lo que se ha realizado, además involucra la afectividad, actualmente es la definición que reposa en el documento de los estándares curriculares en Colombia, emanados por el Ministerio de Educación Nacional MEN.

"Lo fundamental del trabajo orientado al desarrollo competencial del alumnado es que, ante una situación contextualizada o no, este se sabe enfrentar a la misma con las herramientas matemáticas que posee. No vamos a reconocer si se sabe resolver ecuaciones, sino si se sabe usar ecuaciones para resolver un problema. Sol, Jimenez y Rosich (2007),"7

Finalmente una concepción hacia la efectividad a la hora de solucionar problemas. Se acepta la competencia si la acción muestra como evidencia la solución de un problema.

\section{Relación entre la teoría de los campos conceptuales $\mathrm{CC}$ y el modelo re-descripción representacional RR en la Enseñanza de las ciencias.}

Partimos del supuesto que el objetivo primordial de la escuela es el tratamiento de los conceptos científicos, como lo propone Vergnaud,

La question du développement des concepts scientifiques chez l'enfant d'âge scolaire est avant tout une question pratique d'uneimportance immense, peut être même primordiale pour les problèmes que pose à l'école l'enseignement du système de connaissances scientifiques. Or ce que nous savons sur cette question frappe par son indigence. Sa portée théorique n'est past moins grande, puisque l'étude du développement des concepts scientifiques, c'est-à-diredes concepts authentiques, incontestables, des vrais concepts, ne peut manquer de mettre en lumière les règles les plus profondes, essentielles, qui sont au fondement de tout processus de formation des concepts en général. (Vergnaud: 2004. Pág 11).

\footnotetext{
${ }^{6}$ ESTÁNDARES BÁSICOS DE COMPETENCIASEN MÁTEMÁTICAS pag 50

${ }^{7}$ cita de: Competencias básicas: competencia matemática Marzo de 2009 Félix Rodríguez Díaz Universitat de les Illes Balearsfelix.rodriguez@uib.es
} 
Se requiere que la escuela forme propicie el trabajo con conocimientos científicos, partiendo de los conocimientos cotidianos pero no quedándose allí. Es importante para la ciencia y en especial para la enseñanza de la ciencia resaltar que:

- El conocimiento científico y las teorías se soportan sobre supuestos que son sometidos a falsación y adquieren mayor fuerza, cuanto más resistan la falsación, esta posibilidad de falsación es lo que le otorga dinamismo y progreso a la actividad científica.

- Las teorías actualmente aceptadas han superados los intentos de falsación pero no por ello son absolutas y acabadas, además, si aún siguen vigentes puede ser por la falta de "ingenio", de quienes han intentado falsarlas.

- Las teorías científicas, se construyen continuamente y no son estáticas ya que su núcleo fuerte puede ser falsado o contenido por otra teoría más elaborada.

El conocimiento científico necesariamente debe ser verbalizado, porque de modo contrario no daría espacio para la refutación o aceptación al interior de una comunidad científica, la falta de explicación niega la posibilidad de falsación de los argumentos y la oportunidad de establecer conciliaciones teóricas alrededor de una situación. Ahora, la verbalización no es fácil, ni siempre puede lograrse según se observa en la teoría de Karmiloff-Smith, lograr la verbalización, supone un esfuerzo mayor y es lo que permite trascender del conocimiento cotidiano al conocimiento científico.

Desde la TCC, una persona pone en evidencia sus invariantes operatorios al enfrentarse a una situación, los invariantes operatorios, pueden ser inconscientes, es decir, una respuesta "automatizada", de ese primer estado, pasan de ser consientes a explicitables, luego explícitos y finalmente formalizados, tal como lo presenta el siguiente gráfico (Vergnaud, 2007a; 299)

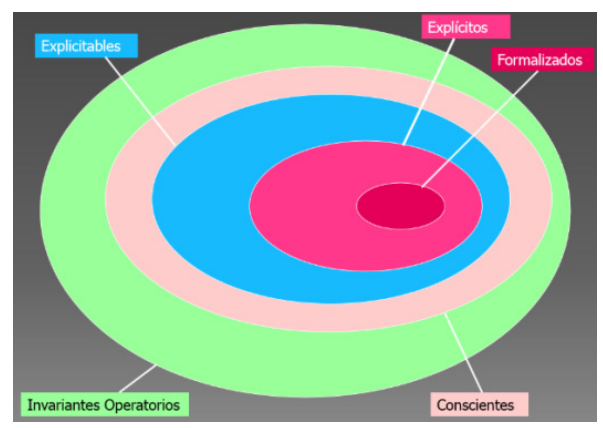

Diagrama invariantes operatorios, conocimiento consciente explicitable, explícito y formalizado. 
El conocimiento cotidiano no llega a la formalización, es decir no puede ser verbalizado. En términos del modelo RR el conocimiento científico las representaciones se encuentran en el dominio E3, se puede verbalizar. Así, esas respuestas automatizadas, pueden ser entendidas como competencias, porque llevas a una acción al interior de una situación, pero no necesariamente pasa del conocimiento cotidiano.

Así, el reto es la enseñanza de conceptos científicos, usando como base los conceptos cotidianos. Un proceso que busca que el concepto sea explícito verbalizado y luego de verbalizado, preferiblemente formalizado. Ahora, al interior de la teoría RR, las representaciones no pueden quedarse en el dominio E2.

El conocimiento cotidiano hace parte del conocimiento previo, que potencialmente puede ser conocimiento explícito con la ayuda de la mediación docente. En este caso es el paso del conocimiento cotidiano (teoremas en acto-conceptos en acto) al conocimiento científico, en representación explicita, verbalizada y en el mejor de los casos formalizada.

En la TCC lo importante es la situación que permite evocar y dar sentido a los conceptos, pero, es necesario reconocer que los individuos poseen un conocimiento previo, que regula sus invariantes operatorios, por tal razón, la labor del docente es analizar las situaciones identificando los conceptos que están en juego y cuáles son las continuidades y rupturas, partiendo del conocimiento previo de los estudiantes, que en este caso se clasifica como conceptos cotidianos, las cuales pueden ser simplemente acciones aprendidas que son eficaces y presentan al individuo como competente. Se acepta ese conocimiento anterior como una oportunidad de trabajo, no como un elemento a desechar, se le otorga especial importancia como conocimiento previo, parte de la experiencia individual, que finalmente, conforma el escenario en el cual han tenido sentido los conceptos aprendidos y los esquemas de los cuales dispone el individuo. Ahora, se requieren elaborar nuevos esquemas que permitan enfrentarse a nuevas situaciones, aunque, lo que consecuentemente requiere nuevos invariantes operatorios. Un individuo en una situación: Primero, puede que tenga un claro un esquema que le permite afrontarla de forma mecánica, y segundo, puede ocurrir que tenga todos los conocimientos para emplear un esquema en particular, sino que deba probar varios y posiblemente conseguir uno nuevo para enfrentar la situación. En los dos casos se puede suponer que el individuo tiene éxito, aunque el tiempo que dure lograrlo no sea corto, lo que puede clasificarlo como competente. Sin 
embargo, no está garantizada la verbalización, al solicitar al individuo que explique sus razonamientos puede ocurrir que no pueda lograrlo, así, aún continúa en plano el conocimiento cotidiano.

\section{La competencia como punto inicial para el proceso de enseñanza aprendizaje.}

En el país se utiliza el termino competencia, definida en pocas palabras como un saber-hacer en contexto, con idoneidad. Competencia indica realizar una actividad eficaz o incluso eficiente, sin embargo, puede realizarse esta actividad sin tener la posibilidad de explicar y formalizar lo que ha ocurrido, los conceptos utilizados o las teorías involucradas, paso importante para evolucionar de los conceptos cotidianos hacia los conceptos científicos. Así, desde la teoría de los campos conceptuales la competencia no es más que el punto de partida para la investigación y la búsqueda de estrategias para lograr la transición de conceptos implícitos a explícitos, con la característica que se puedan verbalizar, ya que esta característica es fundamental para el conocimiento científico y esa verbalización debe lograr, aunque posiblemente no en todos los casos la formalización. La misma situación se tiene desde el modelo RR, la competencia, es el estadio de maestría conductual que se adquiere para estabilizarse y luego realizar una rerepresentación, esta maestría conductual se alcanza en los niveles E1 y E2, pero no se tiene certeza que pasará al nivel E3, de verbalización, ya que, ser competente no implica explicitar lo que se ha realizado.

En conclusión, la enseñanza por competencias puede quedarse en el saber cotidiano, sin dar paso a procesos explícitos verbalizados en busca de formalización. Aunque, como saber previo es valioso, la competencia es apenas un punto de partida (teoremas en acto, conceptos en acto, dominio E2) para lo que se pretende en enseñanza en ciencias, según como se infiere desde las teorías neopiagetianas de los $\mathrm{CC}$ y el modelo RR. De esta forma se espera haber justificado la hipótesis inicial: Es un error en la enseñanza en ciencias, considerar la competencia como punto final del proceso educativo, por el contrario, la competencia adquiere la característica de estado inicial en el camino hacia la formación de conocimiento científico. 


\section{Bibliografía}

BITTAR, Marilena y Muniz, Cristiano Alberto (2009): A aprendizagem matemática na perspectiva da teoria dos campos conceituais. Editora CRV. 2009

CHALMERS, A. (1988). ¿Qué es esa cosa llamada Ciencia? Siglo XXI editores. Cap. 4, 5 y 6. Pp. 59 - 109.

KARMILOFF-SMITH, Annette (1994): Más Allá de la modularidad, la ciencia cognitiva desde la perspectiva del desarrollo. Alianza editorial. (1994).

Instituto Colombiano Para El Fomento De La Educación Superior -ICFES (2007). Sobre Las pruebas saber y de estado. Bogotá.

MEN(2006). Estándares Básicos De Competencias en Matemáticas. http://www.mineducacion.gov.co/cvn/1665/article-116042.html Pág. 50

PALMA, H. (2008). Filosofía de las ciencias. Temas y problemas. Cap. 3: "La ciencia como producto (2). El racionalismo crítico de Karl Popper” Pp. 87 -115.

POPPER, K. (1991). Conjeturas y Refutaciones. Ed. Paidós. Cap. 10, Pp. 264 - 305.

VERGNAUD, Gérard (1990): La Teoría De Los Campos Conceptuales. Recherches en Didáctique des Mathématiques, Vol. 10,nº 2, 3, pp. 133-170, 1990.

VERGNAUD, Gérard (2004): Lev Vygotski Pédagogue et penseur de notre temps. Editions Hachette Education. (2004).

VERGNAUD, Gérard (2007a): ¿En qué sentido la teoría de los campos conceptuales puede ayudarnos para facilitar aprendizaje significativo? (In what sense the conceptual fields theory might help us to facilitate meaningful learning). Investigações em Ensino de Ciências - V12(2), pp.285-302, 2007.

VERGNAUD, Gérard (2007b): Forma operatoria y forma predicativa del conocimiento. I Encuentro Nacional sobre Enseñanza de la Matemática. Traducción de Maria Rita Otero, Conicet -Niecyt, UNICEN, Tandil, Argentina. 